\title{
CLIMABR Parte II: Geração do perfil de precipitação'
}

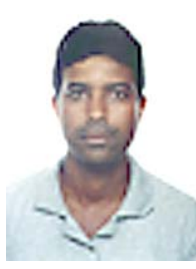

Vicente de P. S. de Oliveira ${ }^{2}$, Sidney S. Zanetti ${ }^{3}$ \& Fernando F. Pruski ${ }^{4}$

\begin{abstract}
${ }^{1}$ Parte da Tese do primeiro autor, apresentada no Programa de Pós-graduação em Engenharia Agrícola na UFV. Viçosa, MG 2 NPGA/CEFET CAMPOS. Rua Dr. Siqueira 273, Parque Dom Bosco, CEP 28030-130, Campos dos Goytacazes, RJ. Fone: (22) 2733-3255, Ramal: 4240. .E-mail: vsantos@cefetcampos.br

${ }^{3}$ UENF/CCTA/LEAG. Av. Alberto Lamego 2000, Pq. Califórnia, CEP 28013-602, Campos dos Goytacazes, RJ. Fone: (22) 2726-1543. E-mail: sidney@uenf.br

${ }^{4}$ UFV/DEA. Campus Universitário, CEP 36571-000, Viçosa, MG. Fone: (31) 3899-1912. E-mail: ffpruski@.ufv.br
\end{abstract}

Protocolo 164 - 6/5/2004 - Aprovado em 4/4/2004

\begin{abstract}
Resumo: As pesquisas sobre precipitação no Brasil têm-se restringido à previsão da lâmina total máxima ou da intensidade máxima provável, esta feita com base em séries históricas. Poucos trabalhos, no entanto, consideram o perfil correspondente a essas precipitações. A falta de modelos representativos tem forçado o uso daqueles desenvolvidos no exterior; contudo, os resultados obtidos demonstram, muitas vezes, que eles não representam adequadamente as condições climáticas típicas do Brasil. Objetivou-se, com o presente trabalho, detalhar complementarmente o desenvolvimento do modelo denominado CLIMABR, descrito por Oliveira et al. (2003), e mostrar sua aplicação para a geração do perfil instantâneo associado às séries sintéticas de precipitação, para as condições climáticas encontradas no Estado do Rio de Janeiro. Na série sintética gerada foram obtidos, para cada dia simulado, entre outros resultados, a duração dos eventos e os valores padronizados da intensidade máxima instantânea de precipitação e do tempo de sua ocorrência ao longo dos eventos. Os dois últimos valores obtidos no modelo foram utilizados para a obtenção do perfil de precipitação representado por uma função dupla exponencial, que foi ajustada a cada evento diário.
\end{abstract}

Palavras-chave: chuva, modelo hidrológico, gerador climático

\section{CLIMABR PART II: Generation of precipitation profile}

\begin{abstract}
The research about precipitation in Brazil has been restricted, most of time, to predict the maximum - total - or probable maximum intensity, this being done on the basis of historical series. Only few projects consider the corresponding profiles to these precipitations. The lack of representative models has forced the use of models developed abroad, despite the fact that the obtained results demonstrate, many times, was in inadequability to represent the typical climatic conditions in Brazil. The aim of the present work to detail complementarily to the development of the CLIMABR model, described by Oliveira et al. (2003), and show its application for instantaneous profile generation associated to synthetic precipitation series, for the climatic conditions of the State of Rio de Janeiro. In the generated synthetic series were obtained for each simulated day, among other informations, the duration of the events, the standardized values of the instantaneous maximum precipitation and the time of its occurrence throughout the events. The last two values were utilized to get the precipitation profile represented by a double exponential function that was adjusted to each daily event.
\end{abstract}

Key words: rain, hydrologic model, climate generator

\section{INTRODUÇÃO}

Os trabalhos sobre avaliação de perdas de solo e água desenvolvidos no Brasil têm empregado, pela falta de informações, padrões de chuvas obtidos no exterior que, provavelmente, não condizem com os padrões que ocorrem nas diferentes regiões do território brasileiro (Pinto, 1995; Vieira et al., 1998; Freitas et al., 2001).

Flanagan et al. (1988) utilizaram seis padrões diferentes de chuva para estudar os efeitos do perfil de precipitação nas perdas de água, na infiltração e na erosão, sendo que, nos quatro primeiros perfis considerados, os picos ocorreram, 
respectivamente, nos primeiro, segundo, terceiro e quarto quartis, considerados a partir do início da chuva. Foram utilizadas também, neste trabalho, chuvas de intensidade uniforme, já bastante empregadas em trabalhos de simulação, além de um sexto padrão específico proposto pelos pesquisadores. Quando comparada com o padrão uniforme, a chuva intensa com padrão atrasado proporcionou taxa de escoamento superficial, cerca de seis vezes maior e três vezes mais perdas de solo, em que os resultados obtidos indicaram que o perfil das chuvas e a intensidade máxima de precipitação afetam significativamente as taxas de escoamento superficial e as perdas de solo, a níveis de significância de 5 e 1\%, respectivamente.

Utilizando 29 anos de registros pluviométricos com o intuito de fornecer subsídios para o planejamento de pesquisa em conservação de água e solo em Santa Maria, RS, Mehl (2000) caracterizou, em seu estudo, três padrões de intensidade de chuvas: avançado, intermediário e atrasado. Neste trabalho, os padrões de chuva intermediário e atrasado representaram, respectivamente, 22,8 e $20,2 \%$ das chuvas intensas. Segundo Flanagan et al. (1988), são esses padrões que produzem maiores perdas de solo e água devido, provavelmente, à maior umidade do solo no momento de ocorrência das máximas intensidades, o que reduz a capacidade de infiltração de água e favorece a desagregação do solo pelo impacto das gotas e, conseqüentemente, a ocorrência, tanto do selamento como do escoamento superficiais.

Alguns modelos buscam representar os padrões das chuvas naturais através da obtenção do perfil característico da precipitação (Brow \& Foster, 1987; Arnold \& Williams, 1989). Nicks et al. (1995) utilizaram uma função dupla exponencial para representar os padrões de chuvas de ocorrência típica nos Estados Unidos; para utilização no desenvolvimento de um gerador climático denominado CLIGEN, no qual a intensidade da chuva é exponencialmente crescente com o tempo, até atingir a intensidade máxima instantânea de precipitação, a partir deste tempo, decrescente, até o final do evento.

Uma exponencial decrescente, com a intensidade máxima instantânea de precipitação ocorrendo a partir do início do evento, é a característica do modelo proposto por Pruski et al. (1997). Na equação proposta, os parâmetros utilizados foram obtidos a partir da equação de intensidade, duração e freqüência para a localidade em estudo, expressa por:

$$
\mathrm{i}_{\mathrm{i}}=\mathrm{i}_{\mathrm{m}}\left(1-\frac{\mathrm{ct}}{\mathrm{t}+\mathrm{b}}\right)
$$

sendo:

$$
\mathrm{i}_{\mathrm{m}}=\frac{\mathrm{KT}^{\mathrm{a}}}{(\mathrm{t}+\mathrm{b})^{\mathrm{c}}}
$$

em que:

$$
\begin{aligned}
& \mathrm{i}_{\mathrm{i}} \text { - intensidade instantânea de precipitação, } \mathrm{mm} \mathrm{h}^{-1} \\
& \mathrm{i}_{\mathrm{m}} \text { - intensidade máxima média de precipitação, } \mathrm{mm} \mathrm{h}^{-1} \\
& \mathrm{t} \text { - duração da precipitação, min }
\end{aligned}
$$

$\mathrm{T}$ - período de retorno, anos

$\mathrm{K}, \mathrm{a}, \mathrm{b}, \mathrm{c}$ - parâmetros da equação de chuvas intensas relacionadas com a localidade de interesse

Um modelo estocástico simples foi apresentado por Arnold \& Williams (1989) para gerar intensidades máximas de precipitação em $30 \mathrm{~min}$, a partir de dados diários de precipitação. $\mathrm{O}$ padrão avançado de chuva foi identificado como predominante e utilizado como sendo base para o desenvolvimento do modelo, assumindo-se que a intensidade máxima instantânea de precipitação ocorre no início da chuva, corroborando com as premissas de Pruski et al. (1997) e com os resultados obtidos por Mehl (2000). Arnold \& Williams (1989) propuseram estimar a intensidade máxima instantânea de precipitação durante uma chuva, por:

$$
\mathrm{i}_{\mathrm{i}}=-2 \mathrm{R} \ln \left(1-\alpha_{0,5}\right)
$$

em que:

$$
\begin{aligned}
& \mathrm{i}_{\mathrm{i}} \text { - intensidade máxima instantânea de precipitação, } \mathrm{mm} \mathrm{h}^{-1} \\
& \mathrm{R} \text { - precipitação total diária, } \mathrm{mm} \\
& \alpha_{0,5} \text { - parâmetro que expressa a máxima porção do total } \\
& \text { precipitado que ocorre durante } 30 \mathrm{~min}
\end{aligned}
$$

\section{MATERIAL E MÉTODOS}

O modelo desenvolvido, denominado CLIMABR - Gerador de séries sintéticas de precipitação, conforme descrito por Oliveira et al. (2003) permite, além da obtenção de informações sobre a precipitação, a geração do respectivo perfil. A série é gerada de forma que se obtenha, para cada dia chuvoso, apenas informações sobre a precipitação, mas, também, os parâmetros necessários para a representação do perfil de precipitação instantânea, que são os valores padronizados do tempo decorrido entre o início da chuva até a máxima precipitação instantânea, chamado tempo padronizado de ocorrência da intensidade máxima instantânea de precipitação $\left(\mathrm{t}_{\mathrm{i}}^{*}\right)$, da intensidade máxima instantânea de precipitação padronizada $\left(i_{i}^{*}\right)$ e a duração do evento (D), em horas.

Como já informado por Oliveira et al. (2003) o desenvolvimento do modelo foi possível a partir de informações de 11 estações meteorológicas situadas no Estado do Rio de Janeiro e operadas pela Fundação Superintendência Estadual de Rios e Lagoas - SERLA, e pela Companhia LIGHT - Serviços de Eletricidade S. A. Utilizaram-se dados originários de pluviogramas e de pluviômetros.

\section{Duração dos eventos}

A duração diária dos eventos é estimada com base nas relações constatadas entre as intensidades máxima e média das chuvas das estações consideradas $(\Delta)$ e na geração, pelo modelo, da relação entre os valores do máximo precipitado em 30 min e o total diário $\left(\alpha_{0,5}\right)$. A duração das chuvas, por sua vez, foi avaliada conforme proposto por Yu (2001), considerandose o valor do parâmetro $\Delta$ e a precipitação máxima em 30 min $\left(\alpha_{0,5}\right)$ por: 


$$
\mathrm{D}=-\frac{\Delta}{2 \ln \left(1-\alpha_{0,5}\right)}
$$

em que:

D - duração efetiva do evento, $\mathrm{h}$

$\alpha_{0,5}$ - razão entre a precipitação máxima ocorrida em 30 min e a precipitação total diária, adimensional

$\Delta$ - parâmetro que expressa a relação entre os valores diários de intensidade máxima instantânea de precipitação e de intensidade média, adimensional

Visando-se à utilização do CLIMABR em localidades em que só existam dados pluviométricos disponíveis, analisou-se o desempenho do modelo levando-se em conta não só o uso de um valor específico para cada localidade mas, também, o uso de um valor único de $\Delta$ para todas as localidades.

Para a obtenção do parâmetro $\Delta$ gerou-se, de início, um gráfico no qual foram relacionados os valores da intensidade máxima instantânea de precipitação (ordenada) com os da intensidade média (abscissa) para os dias chuvosos observados na série. A intensidade média $\left(\mathrm{i}_{\mathrm{b}}\right.$ ) foi calculada dividindo-se o total precipitado pela duração total do evento, sem a exclusão dos intervalos de tempo durante o evento em que não houve precipitação. Uma equação linear interceptando a origem $(0,0)$ foi ajustada aos pontos, sendo o coeficiente angular desta reta correspondente ao valor do parâmetro $\Delta$.

Os valores de $\alpha_{0,5}$ foram mensurados a partir da razão entre a precipitação máxima ocorrida em 30 min e a precipitação total diária sendo, então, ajustados à distribuição Gama de probabilidade aos valores de $\alpha_{0,5}$, que, segundo Sediyama et al. (1978), se ajusta muito bem a variáveis aleatórias contínuas em climatologia. Para se verificar se a distribuição probabilística representava adequadamente o conjunto de dados observados, aplicaram-se os testes de aderência de Qui-Quadrado $\left(\chi^{2}\right)$ e de Kolmogorov-Smirnov, respectivamente, em níveis de 5 e $20 \%$ de significância.

O procedimento para a geração dos valores de $\alpha_{0.5}$ para os dias chuvosos segue a proposta de Sediyama et al. (1978), cuja metodologia consiste na geração de valores de $\alpha_{0,5}$ para os dias chuvosos, a partir do conhecimento da probabilidade de ocorrência dos eventos de chuva e dos valores dos parâmetros $\alpha$ e $\beta$ da função de densidade probabilística Gama. Este procedimento está representado na Figura 1. Com o valor da probabilidade de ocorrência na ordenada igual ao número aleatório, calcula-se o valor de $\alpha_{0,5}$ a partir do conhecimento da função ajustada e dos seus parâmetros, conforme a indicação apresentada na Figura 1.

\section{Intensidade máxima instantânea de precipitação}

A intensidade máxima instantânea de precipitação foi gerada pelo CLIMABR, através de uma equação potencial obtida por regressão originada de dados de intensidade máxima instantânea e média, observadas nas estações. Para o cálculo da intensidade máxima instantânea de precipitação, utilizou-se uma equação ajustada do tipo potencial, em que a variável independente dessa equação foi obtida da razão entre a precipitação total diária e a duração efetiva da chuva (Figura 2).

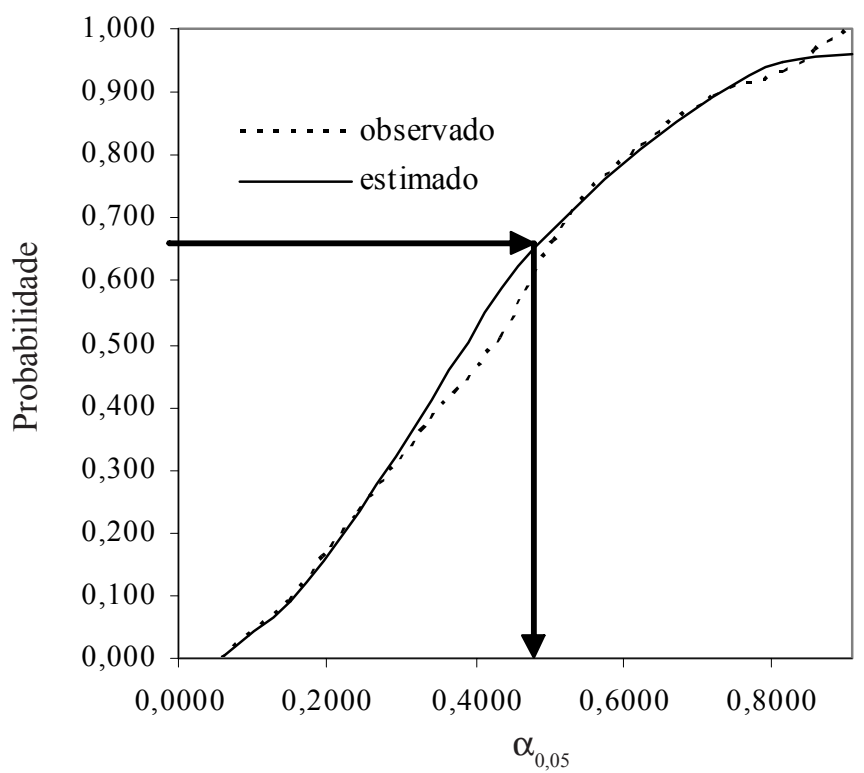

Figura 1. Procedimento para a geração de um valor aleatório de $\alpha_{0,5}$ a partir de uma distribuição de probabilidade

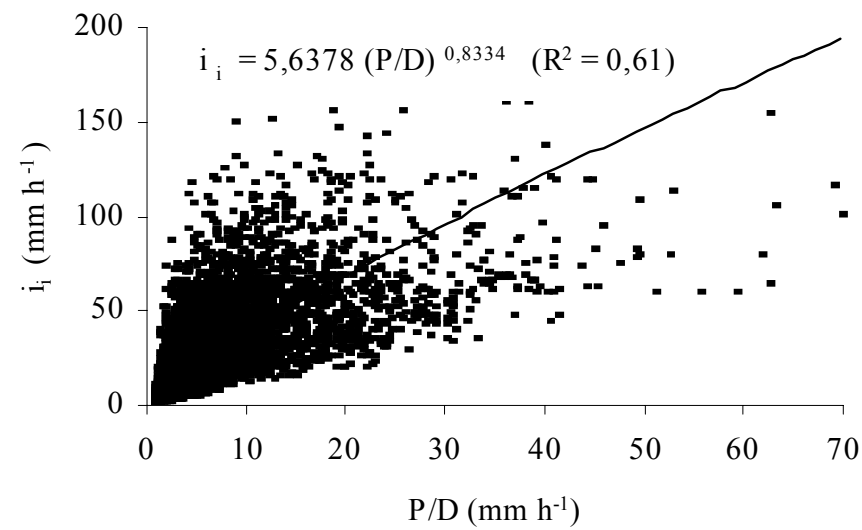

Figura 2. Representação gráfica do procedimento para obtenção da equação potencial utilizada na geração da intensidade máxima instantânea de precipitação

Nota-se que a maior concentração de pontos ocorre próximo à origem do gráfico caracterizando a predominância de eventos de baixa intensidade de precipitação. $\mathrm{O}$ valor do coeficiente de determinação de 0,61 é conseqüência da dispersão dos dados observados nas estações, enquanto a geração do valor padronizado da intensidade máxima instantânea de precipitação é obtida pela razão entre a intensidade máxima instantânea de precipitação e a intensidade média de precipitação gerada para o dia; por sua vez, a intensidade média é obtida pela razão entre a precipitação total diária e a duração efetiva dos eventos, também gerados pelo modelo CLIMABR.

\section{Tempo padronizado de ocorrência da intensidade máxima instantânea de precipitação}

A obtenção do tempo compreendido entre o início do evento de chuva até o tempo de ocorrência da intensidade máxima instantânea de precipitação, se deu através de estudo de freqüência dos valores obtidos na série histórica considerada. Conforme metodologia proposta por Nicks et al. (1995), o 
CLIMABR gerou, inicial e internamente, doze intervalos de classes de mesma amplitude, variando de 0,0 a 1,0, que foram: 0,0 - 0,0833; 0,0833 - 0,1667; 0,1667 - 0,25; 0,25 - 0,3333; 0,3333 0,$4167 ; 0,4167-0,5 ; 0,5-0,5833 ; 0,5833-0,6667 ; 0,6667-0,75$; $0,75-0,8333 ; 0,8333$ - 0,9167; e 0,9167 - 1,0.

O procedimento utilizado consiste na geração de dois novos conjuntos de números aleatórios pelo modelo, que são mantidos inalterados no processamento, permitindo assim a replicação dos resultados experimentais. O primeiro conjunto define a classe onde ocorrerá o tempo de ocorrência da intensidade máxima instantânea de precipitação, sendo o valor do número aleatório igual à freqüência acumulada; o segundo é utilizado no cálculo do valor do tempo de ocorrência da intensidade máxima instantânea de precipitação dentro da classe, através de interpolação obtida pelo resultado do produto entre o número aleatório e a amplitude da classe. $\mathrm{O}$ valor do tempo de ocorrência da intensidade máxima instantânea de precipitação é igual à soma do valor interpolado como o limite inferior da classe. A Tabela 1 exemplifica numericamente o método utilizado.

\section{Perfil de precipitação}

O perfil instantâneo de precipitação foi obtido individualmente para cada evento utilizando-se, para este fim, uma função dupla exponencial (Figura 3). A função dupla exponencial consiste numa exponencial crescente do início do evento até o momento em que ocorre a intensidade de precipitação máxima e, a partir deste momento, uma outra exponencial decrescente descreve o comportamento do perfil até o fim do evento. As informações necessárias para se ajustar este perfil são a intensidade máxima instantânea de precipitação padronizada $\left(i_{i}^{*}\right)$ e o tempo padronizado de ocorrência da intensidade máxima instantânea de precipitação $\left(\mathrm{t}_{\mathrm{i}}^{*}\right)$.

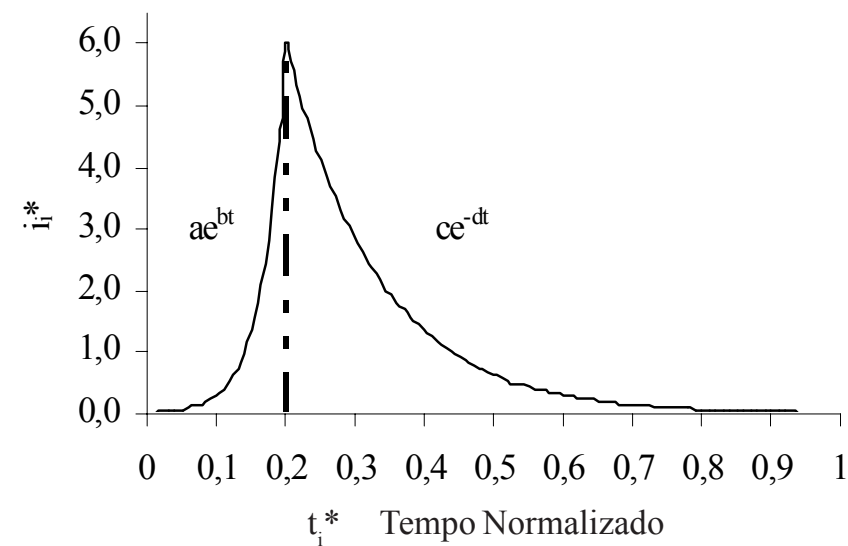

Figura 3. Representação gráfica da função dupla exponencial e das variáveis a ela associadas

Matematicamente, a função dupla exponencial (Nicks et al., 1995) é expressa pelas seguintes equações:

$$
\mathrm{i}(\mathrm{t})= \begin{cases}\mathrm{a} \mathrm{e}^{\mathrm{bt}} & 0 \leq \mathrm{t} \leq \mathrm{tp} \\ \mathrm{ce} \mathrm{e}^{-\mathrm{dt}} & \mathrm{tp}<\mathrm{t} \leq 1,0\end{cases}
$$

em que:

$\mathrm{i}(\mathrm{t}) \quad$ - intensidade padronizada, decimal

a, b, c, d - parâmetros do modelo

$\mathrm{t}_{\mathrm{p}} \quad$ - tempo de ocorrência da intensidade máxima instantânea de precipitação

t $\quad$ - intervalo de tempo

\section{Análise do desempenho do modelo}

Para possibilitar uma avaliação qualitativa e quantitativa do desempenho do CLIMABR, realizaram-se análises comparativas entre a metodologia proposta nesta pesquisa em relação aos dados observados e ao modelo CLIGEN (Nicks et al., 1995), que foi utilizado como referência neste trabalho, para fins de caracterização do desempenho do modelo desenvolvido.

Foram calculadas, também, as variações percentuais $(\delta)$ entre as médias mensais geradas pelos modelos e os valores constatados nas estações. A partir do módulo dos valores das variações percentuais $(\delta)$ calculou-se, em cada estação, o valor médio das variações percentuais (VMVP), o que permitiu a comparação entre os valores gerados pelos modelos CLIMABR e CLIGEN e as estações estudadas.

\section{RESULTADOS E DISCUSSÃO}

\section{Geração dos parâmetros necessários para a estimativa da duração efetiva da precipitação}

A Figura 4 apresenta o gráfico de determinação do parâmetro geral, utilizado na Eq. 4 para cálculo das durações efetivas dos eventos diários de precipitação pelo CLIMABR.

Na obtenção do parâmetro $\Delta$ geral, foram utilizados dados observados em todas as estações estudadas. Nota-se que valores relativos à intensidade máxima instantânea (ordenada)

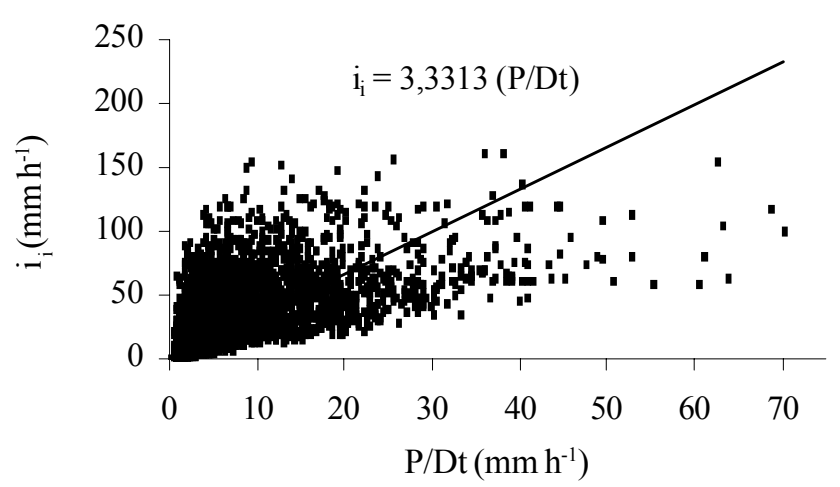

Figura 4. Gráfico de determinação do parâmetro $\Delta$ geral

Tabela 1. Procedimento para a geração do tempo normalizado de ocorrência da intensidade máxima instantânea de precipitação

\begin{tabular}{cccccc|cccccccccc}
\hline $\begin{array}{c}\text { Distribuição } \\
\text { Acumulada }\end{array}$ & 0,652 & 0,746 & 0,789 & 0,830 & 0,845 & 0,857 & 0,873 & 0,893 & 0,903 & 0,930 & 0,961 & 1,000 \\
\hline $\begin{array}{c}\text { Classes } \\
\text { de t }^{*}\end{array}$ & 0 & 0,0833 & 0,1677 & 0,2500 & $\begin{array}{c}0,3333 \\
(0,358)\end{array}$ & \begin{tabular}{c}
0,4167 \\
\hline
\end{tabular}
\end{tabular}


e intensidade média (abscissa) dos eventos de precipitação observados, se concentram próximos à origem, indicando a predominância de eventos de baixa intensidade; entretanto, também podem ser identificados eventos com valores de intensidade máxima instantânea de precipitação acima de $150 \mathrm{~mm} \mathrm{~h}^{-1}$.

Os valores obtidos para os parâmetros $\Delta, \alpha, \beta e \Gamma(\alpha)$ gerais e específicos de cada estação, encontram-se na Tabela 2 , na qual se observam variações nos valores dos parâmetros. A estação Rio Mole apresentou valor do parâmetro $\Delta$ superior às demais estações, enquanto a estação Santa Cecília, os maiores valores dos parâmetros $\alpha$ e $\Gamma(\alpha)$ e a Escola União, a maior estimativa de $\beta$. Os parâmetros $\alpha, \beta$ e $\Gamma(\alpha)$ são usados na geração dos valores de $\alpha_{0,5}$ a partir da função de densidade de probabilidade Gama e, junto com o parâmetro $\Delta$, são empregados no cálculo da duração efetiva dos eventos diários de precipitação, sendo que estes são obtidos a partir de dados pluviográficos.

Tabela 2. Valores dos parâmetros $\Delta, \alpha, \beta$ e $\Gamma(\alpha)$, necessários para a estimativa da duração efetiva da precipitação, considerando-se cada estação individualmente e os valores gerais para todas as estações

\begin{tabular}{lcccc}
\multicolumn{1}{c}{ Estação } & $\Delta$ & $\alpha$ & $\beta$ & $\Gamma(\alpha)$ \\
\hline Cachoeiras de Macacu & 3,218 & 2,55641 & 0,14081 & 1,38420 \\
Capela Mayrink & 3,206 & 2,28020 & 0,16769 & 1,15305 \\
ELETROBRÁs & 3,081 & 2,47041 & 0,14410 & 1,30225 \\
Escola União & 3,290 & 2,03010 & 0,17302 & 1,01310 \\
Ilha dos Pombos & 3,517 & 2,34877 & 0,15565 & 1,20213 \\
Posto Garrafão & 3,086 & 2,01560 & 0,16342 & 1,00669 \\
Rio Mole & 4,048 & 2,19195 & 0,13110 & 1,09701 \\
Santa Cecília & 3,365 & 3,44059 & 0,13451 & 3,11435 \\
Tanguá & 3,441 & 2,91674 & 0,14561 & 1,85466 \\
Tocos & 3,178 & 2,50427 & 0,15740 & 1,33334 \\
Lajes & 3,602 & 3,14264 & 0,14329 & 2,29037 \\
Geral & 3,331 & 2,32501 & 0,16035 & 1,18455 \\
\hline
\end{tabular}

Duração efetiva dos eventos diários de precipitação, considerando-se parâmetros gerais

Os valores da duração efetiva dos eventos diários de precipitação observados para a estação Santa Cecília e os dados gerados pelo CLIMABR e pelo CLIGEN, são apresentados na Tabela 3. Pela comparação dos resultados gerados pelo CLIMABR com os dados observados, vê-se que em julho a média mensal gerada superestimou os dados observados em $10,7 \%$ e, em setembro, a média mensal constatada foi subestimada em 40,2\%. Com relação aos desvios-padrão, em julho se observou uma superestimativa igual a $78,1 \%$, seguida do mês de novembro, também com superestimativa de $35,7 \%$. O VMVP das médias mensais foi igual a $14,8 \%$ e, para o desvio padrão, o valor foi de $24,8 \%$.

Nesta mesma estação, o CLIGEN apresentou desempenho inferior ao do modelo CLIMABR com relação às médias mensais calculadas e aos desvios-padrão estimados; em todos os meses em relação aos resultados gerados pelo CLIGEN para a média mensal da duração efetiva dos eventos diários de precipitação, foram inferiores aos estimados pelo CLIMABR. Todos os valores para a média mensal e desvio padrão foram subestimados pelo CLIGEN.

Comparando-se os resultados obtidos pelos modelos, percebe-se claramente que o CLIMABR obteve estimativas superiores em relação aos dados simulados pelo CLIGEN; entretanto, os resultados obtidos pelo CLIMABR apresentaram grandes variações percentuais, quando comparados com os resultados observados, indicando a necessidade do aperfeiçoamento da metodologia para estimativa da duração efetiva dos eventos diários de precipitação.

Arnold \& Williams (1989) estimaram a duração dos eventos de chuva em cinco localidades dos Estados Unidos, a partir de uma distribuição triangular e dos valores de $\alpha_{0,5}$, para as quais foram calculados as médias e os respectivos desvios-padrão. Para Safford, Estado do Arizona, a média mensal foi superestimada em $89,0 \%$ em maio, enquanto em dezembro os valores simulados superaram, em $87,0 \%$, os dados observados.

Tabela 3. Valores médios mensais da duração efetiva dos eventos diários de precipitação $(\overline{\mathrm{D}})$ e respectivos desvios-padrão $(\mathrm{s})$ dos dados observados e gerados pelo CLIMABR e pelo CLIGEN, variações percentuais $(\delta)$ em relação aos dados constatados e valor médio das variações percentuais (VMVP) para a estação Santa Cecília

\begin{tabular}{|c|c|c|c|c|c|c|c|c|c|c|}
\hline \multirow[b]{2}{*}{ Meses } & \multicolumn{2}{|c|}{ Observado } & \multicolumn{4}{|c|}{ CLIMABR } & \multicolumn{4}{|c|}{ CLIGEN } \\
\hline & $\overline{\mathrm{D}}(\mathrm{h})$ & $\mathrm{s}(\mathrm{h})$ & $\overline{\mathrm{D}}(\mathrm{h})$ & $\delta(\%)$ & $\mathrm{s}(\mathrm{h})$ & $\delta(\%)$ & $\overline{\mathrm{D}}(\mathrm{h})$ & $\delta(\%)$ & $\mathrm{s}(\mathrm{h})$ & $\delta(\%)$ \\
\hline Jan & 5,8 & 4,6 & 6,1 & 5,2 & 5,7 & 23,9 & 5,4 & $-6,9$ & 2,5 & $-45,7$ \\
\hline Fev & 5,8 & 5,0 & 6,2 & 6,9 & 5,6 & 12,0 & 4,8 & $-17,2$ & 2,4 & $-52,0$ \\
\hline Mar & 7,3 & 4,9 & 6,1 & $-16,4$ & 5,5 & 12,2 & 4,3 & $-41,1$ & 2,1 & $-57,1$ \\
\hline Abr & 6,7 & 4,4 & 6,1 & $-9,0$ & 5,7 & 29,5 & 3,0 & $-55,2$ & 1,7 & $-61,4$ \\
\hline Mai & 5,7 & 4,7 & 6,3 & 10,5 & 5,6 & 19,1 & 2,1 & $-63,2$ & 1,2 & $-74,5$ \\
\hline Jun & 6,9 & 4,8 & 5,7 & $-17,4$ & 5,2 & 8,3 & 1,7 & $-75,4$ & 1,3 & $-72,9$ \\
\hline Jul & 5,6 & 3,2 & 6,2 & 10,7 & 5,7 & 78,1 & 2,1 & $-62,5$ & 1,2 & $-62,5$ \\
\hline Ago & 8,0 & 4,4 & 6,4 & $-20,0$ & 5,8 & 31,8 & 2,4 & $-70,0$ & 1,4 & $-68,2$ \\
\hline Set & 10,7 & 4,8 & 6,4 & $-40,2$ & 5,5 & 14,6 & 3,8 & $-64,5$ & 2,0 & $-58,3$ \\
\hline Out & 6,3 & 4,2 & 6,2 & $-1,6$ & 5,7 & 35,7 & 4,0 & $-36,5$ & 2,2 & $-47,6$ \\
\hline Nov & 7,8 & 5,5 & 6,1 & $-21,8$ & 5,5 & 0,0 & 4,4 & $-43,6$ & 2,2 & $-60,0$ \\
\hline Dez & 7,2 & 4,0 & 5,9 & $-18,1$ & 5,3 & 32,5 & 4,6 & $-36,1$ & 2,2 & $-45,0$ \\
\hline VMVP & & & & 14,8 & & 24,8 & & 47,7 & & 58,8 \\
\hline
\end{tabular}


Considerando-se o desvio padrão médio mensal em fevereiro, constata-se uma superestimativa de $147,0 \%$ dos valores observados em Reynolds, Estado de Idaho, e 123,0\% em Safford.

\section{Duração efetiva dos eventos diários de precipitação considerando-se parâmetros gerais e específicos}

Os resultados das médias mensais e dos respectivos desvios-padrão da duração efetiva dos eventos diários de precipitação gerados pelo CLIMABR, considerando-se valores dos parâmetros $\Delta, \alpha, \beta$ e $\Gamma(\alpha)$ específicos para cada estação, bem como as variações percentuais em relação aos dados observados e o valor médio dessas variações em relação ao uso dos valores gerais desses parâmetros, encontram-se na Tabela 4.

Tabela 4. Valores médios mensais da duração efetiva dos eventos diários de precipitação $(\overline{\mathrm{D}})$ e respectivos desviospadrão (s) dos dados gerados pelo CLIMABR, utilizando-se os parâmetros $\Delta, \alpha, \beta$ e $\Gamma(\alpha)$ gerais e específicos, variações percentuais $(\delta)$ e o valor médio das variações percentuais (VMVP) desses parâmetros para a estação Rio Mole

\begin{tabular}{cccccccc}
\hline & \multicolumn{2}{c}{ PG* $^{*}$} & & \multicolumn{4}{c}{ Parâmetros Específicos da Estação } \\
\cline { 2 - 3 } \cline { 6 - 8 } Meses & $\overline{\mathrm{D}}(\mathrm{h})$ & $\mathrm{s}(\mathrm{h})$ & & $\overline{\mathrm{D}}(\mathrm{h})$ & $\delta(\%)$ & $\mathrm{s}(\mathrm{h})$ & $\delta(\%)$ \\
\hline Jan & 6,0 & 5,7 & & 6,4 & 6,7 & 5,8 & 1,8 \\
Fev & 6,1 & 5,5 & & 6,5 & 6,6 & 5,7 & 3,6 \\
Mar & 6,1 & 5,6 & & 6,5 & 6,6 & 5,8 & 3,6 \\
Abr & 6,1 & 5,7 & & 6,5 & 6,6 & 5,9 & 3,5 \\
Mai & 6,4 & 5,6 & & 6,8 & 6,2 & 5,8 & 3,6 \\
Jun & 5,9 & 5,4 & & 6,3 & 6,8 & 5,6 & 3,7 \\
Jul & 6,1 & 5,5 & & 6,5 & 6,6 & 5,7 & 3,6 \\
Ago & 6,5 & 6,0 & & 6,9 & 6,2 & 6,1 & 1,7 \\
Set & 6,3 & 5,5 & & 6,7 & 6,3 & 5,7 & 3,6 \\
Out & 6,1 & 5,6 & & 6,5 & 6,6 & 5,7 & 1,8 \\
Nov & 6,1 & 5,4 & 6,5 & 6,6 & 5,6 & 3,7 \\
Dez & 5,9 & 5,2 & 6,3 & 6,8 & 5,4 & 3,8 \\
VMVP & & & & 6,5 & & 3,2 \\
\hline
\end{tabular}

De maneira geral, observa-se que o CLIMABR apresentou pequenas variações percentuais quando foram geradas as durações efetivas a partir dos parâmetros específicos das estações, comparados com os valores obtidos através dos valores gerais desses parâmetros, tanto para as médias mensais quanto para os desvios-padrão.

Levando-se em conta os valores médios das variações percentuais (VMVP) entre as durações efetivas dos eventos diários de precipitação a partir do uso de parâmetros gerais e específicos no CLIMABR, observa-se que o VMVP da média mensal foi, nesta estação, igual a $6,5 \%$ e, para o desvio padrão, de 3,2\%. O valor máximo de VMVP para as médias mensais ocorreu na estação Lajes, alcançando $23,2 \%$ e, para o desvio padrão, o valor foi igual a $23,3 \%$ na mesma estação. Verificouse que os parâmetros específicos $\alpha$ e $\Gamma(\alpha)$ pertencentes à estação de Lajes utilizados na obtenção de $\alpha_{0,5}$, são superiores aos valores dos parâmetros gerais, o que pode explicar o maior valor de VMVP e as subestimativas geradas, tanto para as médias mensais como para os desvios-padrão. A estação Santa Cecília apresentou comportamento semelhante ao da estação de Lajes com relação às variações percentuais, onde também foram observados valores dos parâmetros $\alpha$ e $\Gamma(\alpha)$ superiores aos parâmetros gerais.

Avaliando-se os resultados obtidos, constatou-se que o modelo CLIMABR apresentou variações nos resultados gerados para as durações efetivas dos eventos diários de precipitação, quando estas foram calculadas a partir dos parâmetros específicos das estações, em comparação com os valores obtidos utilizando-se parâmetros gerais. O uso de valores diferenciados para esses parâmetros proporciona variações percentuais nos resultados da duração efetiva no CLIMABR; entretanto, o emprego de valores comuns desses parâmetros permite a obtenção desta variável na região estudada, em estações que só disponham de dados pluviométricos, o que justificaria o seu uso.

Intensidade máxima instantânea de precipitação padronizada Intensidade máxima instantânea de precipitação, considerandose parâmetros gerais: A intensidade máxima instantânea de precipitação foi padronizada para cada evento diário de precipitação pela intensidade média que, por sua vez, foi obtida pela razão entre a precipitação total diária e a duração efetiva; portanto, os parâmetros $\Delta, \alpha, \beta$ e $\Gamma(\alpha)$ influenciam também os valores gerados da intensidade instantânea máxima de precipitação padronizada, sendo que, neste caso, foram utilizados os parâmetros gerais. A Tabela 5 apresenta os resultados gerados para a estação Tanguá.

Com relação aos resultados gerados pelo CLIMABR nesta estação, as médias mensais apresentaram variações de $-12,5$ (novembro) a 35,9\% (outubro), sendo que na estação Santa Cecília, no mês de julho esta variação alcançou 82,9\%. Os desvios-padrão apresentaram variações percentuais que oscilaram entre os valores de $-44,4 \%$ (julho) e $-86,5 \%$ (dezembro), enquanto as estações Posto Garrafão, Cachoeiras de Macacu e Ilha dos Pombos, apresentaram também grandes variações percentuais.

As variações percentuais entre os resultados obtidos utilizando-se o CLIGEN e os valores observados, apresentaram maior amplitude, variando de $-63,0 \%$ (dezembro) a $25,6 \%$ (outubro), em que as estações de Lajes (-75,7\% em janeiro) e Santa Cecília (77,1\% em julho) também mostraram grandes variações.

Os desvios-padrão obtidos pelo CLIGEN indicaram variações percentuais em relação aos dados observados (Tabela 5), que oscilaram entre -55,8\% (dezembro) e 122,2\% (julho). Nas demais estações constataram-se resultados semelhantes.

Comparando-se os resultados obtidos pelos modelos, percebe-se claramente que tanto o CLIMABR quanto o CLIGEN geraram dados com grandes variações percentuais em relação aos dados observados. Os resultados obtidos indicam a necessidade do aperfeiçoamento da metodologia para a estimativa da intensidade máxima instantânea de precipitação.

Intensidade máxima instantânea de precipitação padronizada considerando-se parâmetros gerais e específicos: Os resultados das médias mensais e dos respectivos desviospadrão da intensidade máxima instantânea de precipitação 
Tabela 5. Valores médios mensais da intensidade máxima instantânea de precipitação padronizada $\left(\overline{i_{i}{ }^{*}}\right)$ e os respectivos desviospadrão (s) dos dados observados e gerados pelos CLIMABR e CLIGEN, variações percentuais $(\delta)$ em relação aos dados observados e valor médio das variações percentuais (VMVP) para a estação Tanguá

\begin{tabular}{|c|c|c|c|c|c|c|c|c|c|c|}
\hline \multirow[b]{2}{*}{ Meses } & \multicolumn{2}{|c|}{ Observado } & \multicolumn{4}{|c|}{ CLIMABR } & \multicolumn{4}{|c|}{ CLIGEN } \\
\hline & $\overline{\mathrm{i}_{\mathrm{i}}{ }^{*}}$ (decimal) & $\mathrm{s}$ (decimal) & $\overline{\mathrm{i}_{\mathrm{i}}{ }^{*}}$ (decimal) & $\delta(\%)$ & s (decimal) & $\delta(\%)$ & $\overline{\mathrm{i}_{\mathrm{i}}^{*}}$ (decimal) & $\delta(\%)$ & $\mathrm{s}($ decimal $)$ & $\delta(\%)$ \\
\hline Jan & 4,8 & 2,9 & 4,9 & 2,1 & 0,8 & $-72,4$ & 1,8 & $-62,5$ & 2,4 & $-17,2$ \\
\hline Fev & 5,5 & 3,3 & 4,9 & $-10,9$ & 0,8 & $-75,8$ & 2,5 & $-54,5$ & 3,0 & $-9,1$ \\
\hline Mar & 5,5 & 3,7 & 4,8 & $-12,7$ & 0,8 & $-78,4$ & 3,5 & $-36,4$ & 3,3 & $-10,8$ \\
\hline Abr & 5,0 & 2,5 & 4,9 & $-2,0$ & 0,9 & $-64,0$ & 4,9 & $-2,0$ & 3,8 & 52,0 \\
\hline Mai & 5,1 & 2,4 & 5,0 & $-2,0$ & 0,8 & $-66,7$ & 4,9 & $-3,9$ & 3,4 & 41,7 \\
\hline Jun & 5,4 & 3,5 & 5,3 & $-1,9$ & 1,0 & $-71,4$ & 5,4 & 0,0 & 4,8 & 37,1 \\
\hline Jul & 4,4 & 1,8 & 5,4 & 22,7 & 1,0 & $-44,4$ & 5,2 & 18,2 & 4,0 & 122,2 \\
\hline Ago & 5,8 & 2,9 & 5,5 & $-5,2$ & 1,0 & $-65,5$ & 5,2 & $-10,3$ & 4,2 & 44,8 \\
\hline Set & 4,5 & 2,6 & 5,4 & 20,0 & 0,9 & $-65,4$ & 4,8 & 6,7 & 3,1 & 19,2 \\
\hline Out & 3,9 & 2,6 & 5,3 & 35,9 & 0,8 & $-69,2$ & 4,9 & 25,6 & 3,8 & 46,2 \\
\hline Nov & 5,6 & 4,1 & 4,9 & $-12,5$ & 0,7 & $-82,9$ & 3,6 & $-35,7$ & 3,2 & $-22,0$ \\
\hline Dez & 5,4 & 5,2 & 4,8 & $-11,1$ & 0,7 & $-86,5$ & 2,0 & $-63,0$ & 2,3 & $-55,8$ \\
\hline VMVP & & & & 11,6 & & 70,2 & & 26,6 & & 39,8 \\
\hline
\end{tabular}

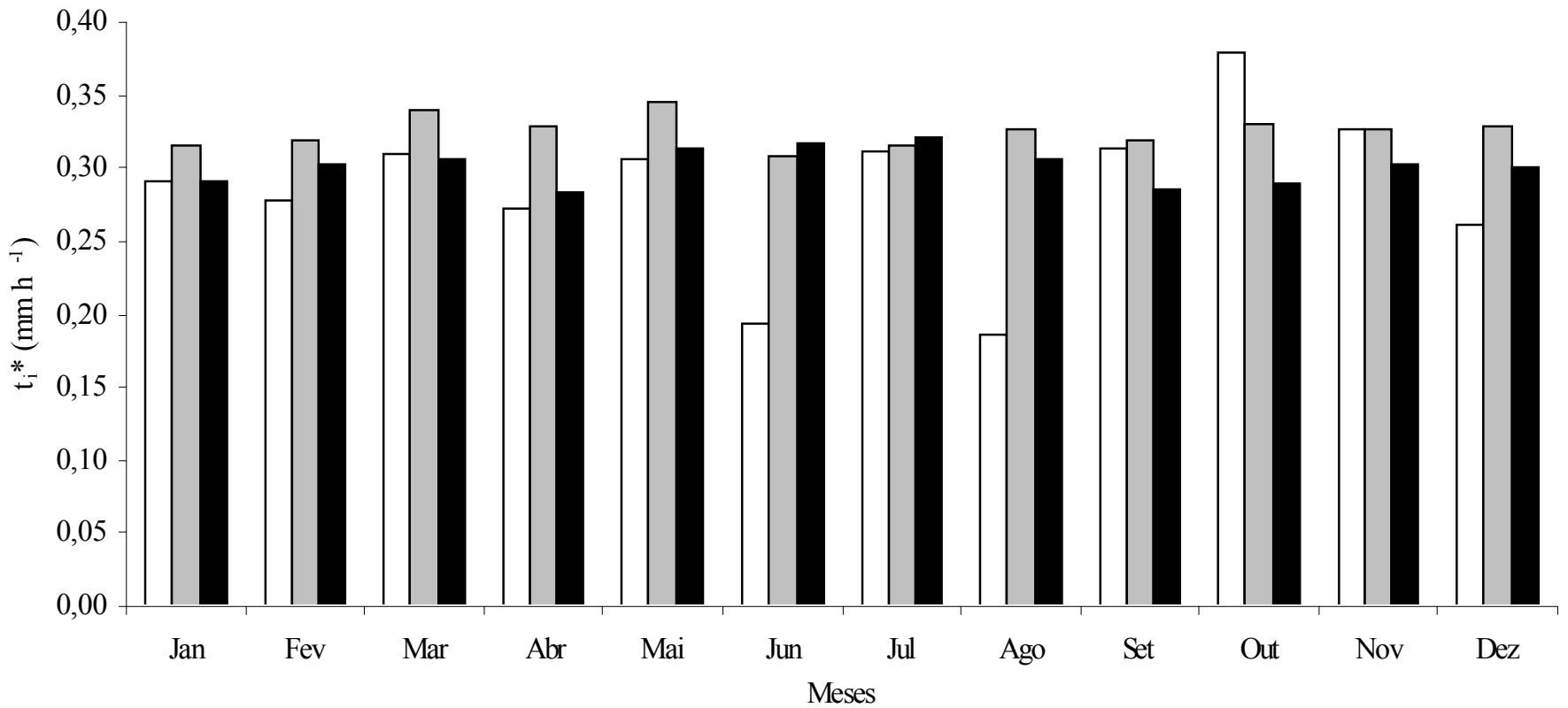

Figura 5. Distribuição mensal do tempo de ocorrência da intensidade máxima instantânea de precipitação padronizado para a estação Santa Cecília

gerados pelo CLIMABR, considerando-se valores dos parâmetros $[\Delta, \alpha, \beta$ e $\Gamma(\alpha)]$ específicos de cada estação, bem como as variações percentuais e o valor médio dessas variações, em relação ao uso de valores gerais desses parâmetros, para a estação Tocos, encontram-se na Tabela 6 .

Observou-se, de maneira geral, que o CLIMABR apresentou pequenas variações percentuais quando foram geradas as intensidades máximas instantâneas a partir dos parâmetros específicos das estações, em comparação com os valores obtidos utilizando-se os valores gerais desses parâmetros.

Os resultados sugerem que o uso de parâmetros gerais para esta variável mostrou-se adequado, indicando a possibilidade de aplicação da metodologia de cálculo para a obtenção da intensidade máxima instantânea de precipitação, mesmo para estações localizadas fora da região de estudo, nas quais só estejam disponíveis dados pluviométricos e que, portanto, não apresentam informações que permitam o cálculo da intensidade máxima instantânea de precipitação padronizada.

Tempo de ocorrência da intensidade máxima instantânea de precipitação padronizado: Os valores observados do tempo padronizado de ocorrência da intensidade máxima instantânea de precipitação para a estação Santa Cecília, bem como os dados gerados pelos CLIMABR e CLIGEN, são apresentados na Figura 5.

Pela comparação dos resultados gerados pelo CLIMABR com os dados observados, verifica-se a ocorrência de variações percentuais diferenciadas em junho e outubro. 
Tabela 6. Valores médios mensais da intensidade máxima instantânea de precipitação padronizada $\left(\overline{i_{i}{ }^{*}}\right)$ e respectivos desvios-padrão (s) dos dados gerados pelo CLIMABR, utilizando-se os parâmetros $\Delta, \alpha, \beta$ e $\Gamma(\alpha)$ gerais e específicos, variações percentuais $(\delta)$ e valor médio das variações percentuais (VMVP) desses parâmetros para a estação de Tocos

\begin{tabular}{|c|c|c|c|c|c|c|}
\hline \multirow[b]{2}{*}{ Meses } & \multicolumn{2}{|c|}{ Parâmetros Gerais } & \multicolumn{4}{|c|}{ Parâmetros Específicos da Estação } \\
\hline & $\begin{array}{c}\overline{\mathrm{i}_{\mathrm{i}}^{*}} \\
\text { (decimal) }\end{array}$ & $\begin{array}{c}\mathrm{S} \\
\text { (decimal) }\end{array}$ & $\begin{array}{c}\overline{\mathrm{i}_{\mathrm{i}}^{*}} \\
\text { (decimal) }\end{array}$ & $\begin{array}{c}\delta \\
(\%)\end{array}$ & $\begin{array}{c}\text { S } \\
\text { (decimal) }\end{array}$ & $\begin{array}{r}\delta \\
(\%) \\
\end{array}$ \\
\hline Jan & 5,9 & 2,3 & 5,8 & $-1,7$ & 2,3 & 0,0 \\
\hline Fev & 5,9 & 2,3 & 5,8 & $-1,7$ & 2,2 & $-4,3$ \\
\hline Mar & 6,1 & 2,4 & 6,0 & $-1,6$ & 2,3 & $-4,2$ \\
\hline Abr & 6,4 & 2,3 & 6,3 & $-1,6$ & 2,3 & 0,0 \\
\hline Mai & 6,6 & 2,1 & 6,4 & $-3,0$ & 2,1 & 0,0 \\
\hline Jun & 6,5 & 2,1 & 6,4 & $-1,5$ & 2,0 & $-4,8$ \\
\hline Jul & 6,5 & 2,1 & 6,3 & $-3,1$ & 2,0 & $-4,8$ \\
\hline Ago & 6,5 & 2,1 & 6,3 & $-3,1$ & 2,1 & 0,0 \\
\hline Set & 6,5 & 2,1 & 6,4 & $-1,5$ & 2,1 & 0,0 \\
\hline Out & 6,4 & 2,2 & 6,3 & $-1,6$ & 2,1 & $-4,5$ \\
\hline Nov & 6,1 & 2,3 & 6,0 & $-1,6$ & 2,2 & $-4,3$ \\
\hline Dez & 6,0 & 2,3 & 5,9 & $-1,7$ & 2,2 & $-4,3$ \\
\hline VMVP & & & & 2,0 & & 2,6 \\
\hline
\end{tabular}

Considerando-se todas as estações estudadas, o CLIMABR gerou médias mensais que apresentaram variações de -23,8 (Rio Mole em setembro) a 63,2\% (Santa Cecília em junho). Os desviospadrão apresentaram variações percentuais que oscilaram entre uma superestimativa de 136,4 (Ilha dos Pombos em julho) a uma subestimativa de 22,9\% (ELETROBRÁS em outubro).

As variações percentuais entre os resultados obtidos utilizando-se o CLIGEN e os valores observados, apresentaram maior amplitude, variando de $-26,3 \%$ (Lajes em setembro) a $80,0 \%$ (Ilha dos Pombos em junho). Os desvios-padrão obtidos por este modelo mostraram variações percentuais em relação aos dados observados, que oscilaram de $-23,5$ até $163,6 \%$, o que também ocorreu na estação Ilha dos Pombos, nos meses de setembro e julho, respectivamente.

Apesar da desvantagem do CLIMABR com relação aos valores do VMVP gerados para as estações, foi o CLIGEN que apresentou maior amplitude das variações percentuais, tanto para as médias mensais como para os desvios-padrão.

\section{CONCLUSÕES}

1. O CLIMABR apresentou desempenho superior ao CLIGEN; entretanto, os dois modelos mostraram grandes variações percentuais em relação aos valores observados para a duração efetiva dos eventos diários de precipitação, sendo que o mesmo comportamento foi também constatado em relação a intensidade máxima instantânea de precipitação.

2. Os valores gerados do tempo de ocorrência da intensidade máxima instantânea de precipitação padronizada, tanto pelo CLIMABR quanto pelo CLIGEN, indicaram consideráveis variações percentuais em relação aos dados observados nas estações.
3. O desempenho obtido pelo CLIMABR foi similar ao obtido pelo CLIGEN, ressaltando-se que as principais limitações se referiram ao seu uso para a representação adequada do perfil de precipitação.

\section{AGRADECIMENTOS}

Ao Centro Federal de Educação Tecnológica de Campos CEFETCAMPOS e ao Parque de Alta Tecnologia Norte Fluminense - TECNORTE, pela oportunidade concedida em prol da realização deste trabalho. À Fundação Superintendência Estadual de Rio e Lagoas - SERLA, e à Companhia LIGHT Serviços de Eletricidade S.A, pela cessão dos dados que permitiram a execução desta pesquisa.

\section{LITERATURA CITADA}

Arnold, J.G.; Williams, J.R. Stochastic generation of internal storm structure at a point. Transactions of the ASAE, St. Joseph, v.32, n.1, p.161-167, 1989.

Brown, L.C., Foster, G.R. Storm erosity using idealized intensity distributions. Transactions of the ASAE, St. Joseph, v.30, n.2,p.379-386, 1987.

Flanagan, D.C.; Foster, G.R.; Moldenhauer, W.C. Storm patterns effect on infiltration, runoff, and erosion. Transactions of the ASAE, St. Joseph, v.31, n.2, p.414-420, 1988.

Freitas, A. J.; Silva, D.D.; Pruski, F.F.; Pinto, F.A. Pereira, S.B.; Gomes Filho, R.R.; Teixeira, A.F.; Baena, L.G.N.; Mello, L.T.A.; Novaes, L.F. Equações de chuvas intensas no Estado de Minas Gerais. Belo Horizonte: Companhia de Saneamento de Minas Gerais; Viçosa: UFV, 2001. 65p.

Mehl, H.U. Caracterização de padrões de chuvas ocorrentes em Santa Maria, RS e sua relação com as perdas de solo e água em entressulcos. Santa Maria: UFSM, 2000. 53p. Dissertação Mestrado

Nicks, A.D.; Lane, L.J.; Gander, G.A. Weather Generator: USDAWater erosion prediction project (WEPP). West Lafayette: USDA-ARS National Soil Erosion Research Laboratory, 1995. cap.2, p.22.

Oliveira, V.P.S.; Zanetti, S.S.; Pruski, F.F. Desenvolvimento de Modelo para a geração de séries sintéticas de precipitação. In: Congresso Brasileiro de Engenharia Agrícola, 32, 2003, Goiânia. Anais...Goiânia: SBEA, 2003. CD Rom

Pinto, F.R.L. Equações de intensidade-duração-freqüência da precipitação para os Estados do Rio de Janeiro e Espírito Santo: estimativa e espacialização. Viçosa: UFV, 1995. 70p. Dissertação Mestrado

Pruski, F.F.; Ferreira, P.A.; Ramos, M.M.; Cecon, P.R. Model to design level terraces. Journal of Irrigation and Drainage Engineering, Lafayete, v.1, p.8-12, 1997.

Sediyama, G.C.; Chancellor, W.J.; Burkhardt, T.H.; Goss, J.R. Simulação de parâmetros climáticos para a época de crescimento das plantas. Revista Ceres, Viçosa, v.25, n. 141, 1978.

Vieira, D.B.; Lombardi Neto, F.; Santos, R.P. Relação entre intensidade, duração e freqüência de chuvas em Mococa. Pesquisa Agropecuária Brasileira, Brasília, v.33, n.8, p.1215$1220,1998$.

Yu, B. Improvement and evaluation of CLIGEN for storm generation. Transactions of the ASAE, St. Joseph, v.43, n.2, p.301-307, 2001. 